\section{Electrical Science \& Engineering}

https://ojs.bilpublishing.com/index.php/ese

\title{
ARTICLE \\ Basic Unit: As a Common Module of Neural Networks
}

\section{Seisuke Yanagawa*}

OptID, Machida, Tokyo, Japan

\begin{tabular}{l}
\hline ARTICLE INFO \\
\hline Article history \\
Receiced: 05 November 2020 \\
Accepted: 30 November 2020 \\
Published Online: 15 April 2021 \\
\hline Keywords: \\
Acceptance and generation of time-series data \\
Context learning \\
Prediction using context \\
Extended DNN \\
Two-way communication between layers
\end{tabular}

\section{Introduction}

No animal has wheels, but we have incorporated rotating mechanisms in various mechanical devices to run faster than horses and powerful than cows. In addition, since computers have speeds and processing amounts that are incomparable to actual neural circuits, pattern recognition technology using neural networks originating from Perceptron can instantly judge huge amounts of data, large commercial outcome have been taken. However, these progress does not always lead to a deep understanding of the intellectual functions of living organisms.

In the next chapter, I will consider the feeding behavior of seeking food while avoiding the dangers that animals in the early stages of evolution have as essential time-series processing. The processing will be realized by combining elemental circuits with the same structure. A neural net-

\begin{abstract}
In this paper, the logic is developed assuming that all parts of the brain are composed of a combination of modules that basically have the same structure. The feeding behavior of searching for food while avoiding the dangers of animals in the early stages of evolution is regarded as the basis of time series data processing. The module that performs the processing is presented by a neural network equipped with a learning function based on Hebb's rule, and is called a basic unit. The basic units are arranged in layers, and the information between the layers is bidirectional. This new neural network is an extension of the traditional neural network that has evolved from pattern recognition. The biggest feature is that in the processing of time series data, the activated part changes according to the context structure inherent in the data, and can be mathematically expressed the method of predicting events from the context of learned behavior and utilizing it in best action.
\end{abstract}

work that has the essential function is presented basing Hebb's law and called Basic Unit ${ }^{[1]}$. A Basic Unit is a collection of circuits that process time-series data and operates as a component of the whole ${ }^{[2],[3]}$. The Basic Units are arranged in layers, and the information between the layers is bidirectional. When the length of time series data is 1 , it is equivalent to a conventional neural network whose main function is pattern recognition. This new neural network can be said to be a neural network whose functions have been extended from pattern recognition to behavior recognition, learning and generation.

The biggest feature is that in the processing of time series data, the activated part changes according to the context structure inherent in the data, and can be mathematically expressed the method of predicting events from the context of learned behavior and utilizing it in best action.

*Corresponding Author:

Seisuke Yanagawa,

OptID, Machida, Tokyo, Japan;

Email: s_yanagawa@jcom.home.ne.jp 


\section{Basic Units and those Layers}

Even animals in the early stages of evolution move in the direction of light and smell in order to survive, prey on them if they judge that they are food, and fight or avoid them if they are dangerous. This level of behavior could be mimicked by junior high school level electronics by combining sensors and logic ICs, and the number of logic elements used may not be much different from the total number of insects or zooplankton neurons. In more evolved animals, even predatory behavior consists of a time series of multiple behaviors. That is, the process is to extend the arm toward the goal, open the palm when approaching the goal, close the palm when touching the goal, grab the goal and bring it closer to one's mouth. This operation can be realized with a circuit that is a slightly developed version of "electronic work at the junior high school level". For example, Figure 1 is a circuit in which the AND gates are connected in series. The four actuators are connected by rotation axis and show the shoulder, upper arm, lower arm, palm, finger muscles and joints from the left. The gate corresponding to the shoulder receives start signal and signal regarding the angle and load from the rotation axis. If the conditions are met, it drives the actuator and sends a signal to the gate corresponding to the upper arm. It continues until the operation of the actuator corresponding to the finger is completed.

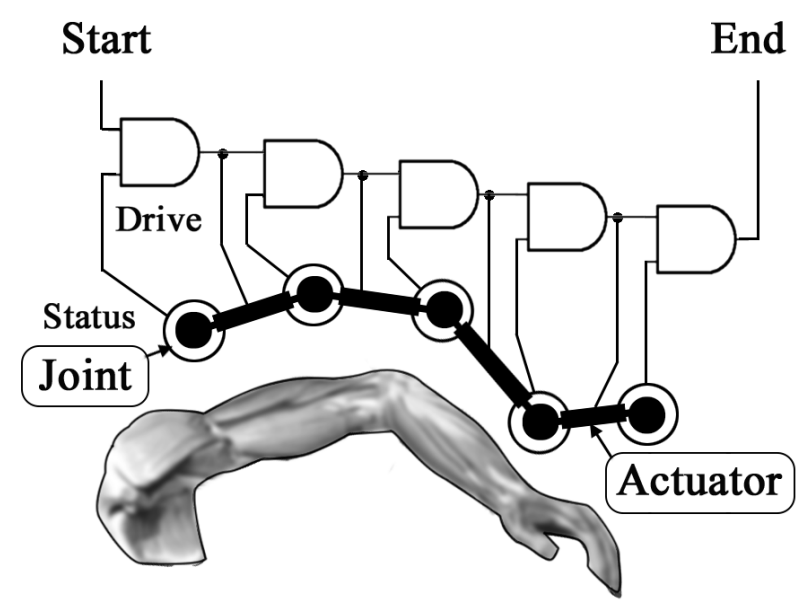

Figure 1. Concept of a circuit that moves the arm

The movement of the arm has various functions such as grasping, hitting, and throwing. Figure 2 shows the circuits arranged by function. It can be said that it is a judgment like choosing a brush that draws the desired picture from the brush stand. It is impossible to develop such a model into a neural network that accepts and generates time series data.

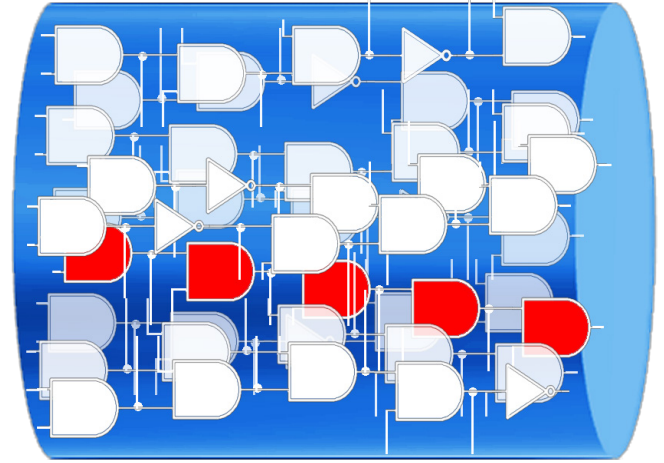

Figure 2. Electronic circuits collected in a bundle

Commercial results may be obtained if calculations based on the similarity of time series data or Markov processes are performed at high speed and in large quantities, but it is far from elucidating the functions of nerve cells in living organisms. Let's think about realizing the function with a model that uses elements that are more familiar with the function of nerve cells, specifically, that have another characteristic according to the Hebb rule.

First, it is shown in Figure 3 that time series data composed of finite type elements can be divided into a plurality of subsequences in which the same element does not occur multiple times. Since the divided subsequences is considered an element of the new time series data in the upper hierarchy, it can be said that every time series data contains a hierarchical context.

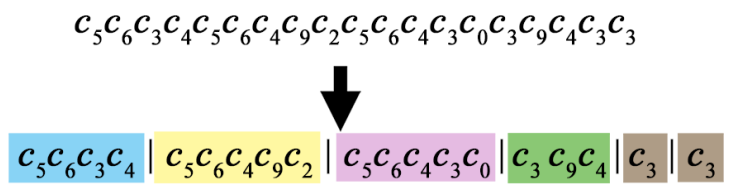

Figure 3. Any string can be split into subsequences

Consider a set $\mathrm{U}$ of elements equivalent to an AND gate as shown in Figure 4 left. Each element is randomly connected and receives the elements of time series data $\mathrm{c}_{0}, \mathrm{c}_{1}$, $c_{2}$ and $c_{3}$ from below. $U_{0}$ is a set of elements activated by receiving the signal $\mathrm{c}_{0}$ as the start of a series of operations. Next, $\mathrm{c}_{1}$ is received following $\mathrm{c}_{0}$, the element with high activity is the set of elements that are already activated by the reception of $c_{0}$. That is, $U_{1}$ is accepts $c_{0} c_{1}$. Similarly the set of highly active elements is narrowed until the last data $c_{3}$ is received, and the last remaining set of elements $\mathrm{U}_{3}$ becomes a pattern showing the time series data $\mathrm{c}_{0} \mathrm{c}_{1} \mathrm{c}_{2} \mathrm{c}_{3}$. Figure 4 Right is an image of a basic unit that processes time series data starting with $\mathrm{c}_{0}$, and this structure may be not so different to the structure of a hypothetical module called mini- and macro-columns, barrels, stripes, or blobs in neuroscience ${ }^{[1]}$. All time-series data can be processed by arranging basic units for the number of elements that 
appear at the beginning of the time-series data and regarding their output as the upper-layer time-series data.

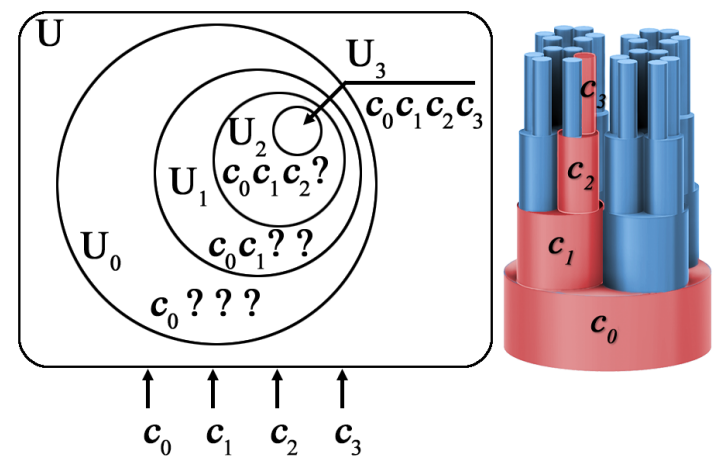

Figure 4. Operation diagram and image of Basic Unit

\section{Conclusions}

The neural network not only controls the current input/ output movement as the hierarchy is added to the upper level, but also stores the history as longer time series data. The context of time series data is represented by a tree-structured graph.

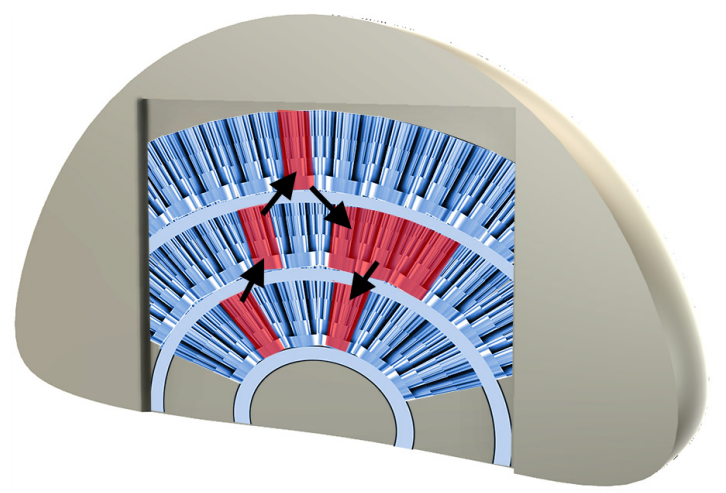

Figure 5. State changing of Basic Units arranged in hierarchal
While the time series data are processing, the activity of the Basic Unit changes along the tree. The next possible states of the Basic Unit located on the leaves of the tree that is connected to the sensory and motor organs follow the tree toward the root, calculate the state of the next activated Basic Unit according to the context. It is obtained by reverse transmission in the tree. In other words, the optimal policy to be taken can be obtained from the calculation by determining in which context the current situation is in the stored time series data. The next evolution of animal intelligence seems to be the acquisition of imitation and symbolization functions ${ }^{[4],[5]}$. The former leads to sociality in a group, and the latter leads to the acquisition of language as a means of communicating information. I hope that this paper will serve as a basis for treating these themes as mathematical objects ${ }^{[6]}$.

\section{References}

[1] G.Buzsaki "Rhythms of the Brain", Oxford university press, 2006, p.43.

[2] S.Yanagawa "Each Role of Short-Term and LongTerm Memory in Neural Networks American Journal of Neural Networks and Applications (AJNNA). (ISSN Print: 2469-7400; ISSN Online: 2469-7419).

[3] S. Yanagawa "From Bacteria, a Consideration of the Evolution of Neural Network" Journal of Mechanics Engineering and Automation 1 (2019) p.17-23.

[4] S.Yanagawa, "Neural Network That Learns Sequential Processing and Predicts by the Context." In The 28th Annual Conference of the Japanese Neural Network Society (JNNS2018).

[5] D. C. Dennet, "From Bacteria to Bach and Back: The Evolution of Minds." In Penguin.

[6] T M.Iacoboni, "Mirroring People." Picador 33. 2008. 